\title{
COMMISSION 27: VARIABLE STARS ${ }^{1}$
}

\author{
(ETOILES VARIABLES)
}

\author{
PRESIDENT: Jørgen Christensen-Dalsgaard \\ VICE-PRESIDENT: Conny Aerts \\ ORGaNIZING COMMITTEE: Carla Cacciari, Peter Cottrell, Ed Guinan, \\ Petr Harmanec, Don Kurtz, Jaymie Matthews, Pawel Moskalik, \\ Dimitar Sasselov, S. Seetha, Doug Welch \& Patricia Whitelock
}

\section{Introduction}

As in the case of the previous report (Transactions, vol. XXIVA), the organizing committee of Commission 27 has decided that an extensive bibliography is unlikely to be of substantial use, given the existing reviews and the easy online availability of literature information. Thus the present report concentrates on some highlights within the field over the previous three years. Further details can be found in the proceedings of the conferences over this period, such as IAU Colloquia No 176 (Budapest, 1999; ASPC No 203) and 185 (Leuven, 2001; ASPC No 259: Aerts et al., 2002), both published by the Astronomical Society of the Pacific, the First Eddington Workshop (Cordoba, 2001; see Favata et al., 2002), the workshop on 'Mass-losing pulsating stars and their circumstellar matter' (Sendai, 2002; proceedings to be published by Kluwer in the ASSL series), the EuroConference on 'Symbiotic stars probing stellar evolution' (La Palma, May 2002; proceedings to be published by ASP), as well as the workshop on 'Asteroseismology across the HR diagram' (Porto, 2002; see Thompson et al., 2002).

\section{New Observational Possibilities}

As highlighted in the previous report, large-scale surveys, looking for light variations caused for example by micro-lensing or exoplanet transits, have generated massive sets of data on a broad variety of variable stars, totally revolutionizing the study of moderate- and largeamplitude variables (see, for example, Alcock et al., 2000; Udalski et al., 1999).

Ground-based observations of small-amplitude oscillators have received a major boost from the development of very stable techniques for Doppler-velocity measurements, primarily motivated by the search for extra-solar planets. As discussed in Section 7 below, this has resulted in the detection of solar-like oscillations in a number of stars.

In view of the extremely small amplitudes expected of solar-like oscillations photometric observations are unlikely to be possible from ground-based observatories, and space observations have been a long-term dream of the asteroseismic community. In addition to photometric stability, space potentially offers the possibility for extended (nearly) continuous observations. The dream became a reality with the realization by Derek Buzasi that the star tracker on the otherwise failed WIRE satellite could be used for very precise observations of oscillations of bright stars. Although the orbit is far from ideal for this purpose, some very interesting results have been obtained (Buzasi et al., 2000; Cuypers et al., 2002). A major breakthrough is expected in the coming few years with the launch of several

${ }^{1} \mathrm{C} 27$ has a homepage at http://www.konkoly.hu/IAUC27/ with a facility for up-dating email addresses. Members are urged to do this. 
space missions, starting with the Canadian MOST mission in 2003 (Matthews et al., 2001) and followed by the French COROT mission (Baglin et al., 2002) and possibly the Danish Rømer mission (Christensen-Dalsgaard, 2002) around 2005 and the Eddington mission of the European Space Agency (Favata, 2002) in 2007-08. These missions will provide data on variability of a very broad range of stars with high sensitivity, in some cases limited only by the intrinsic stellar 'noise' and including numerous stars showing solar-like oscillations.

\section{Large-Amplitude Variables}

Direct interferometric observations, in the near infrared, have been made of the radius variations due to pulsations in selected Cepheids (e.g., Lane et al., 2002). This allows a determination of the distance and mean radius of these stars, as well as a comparison with models of their behaviour. Interferometric determinations of the mean angular diameter of a number of Cepheids have been used to calibrate the so-called Barnes-Evans relation between colour and surface brightness (Nordgren et al., 2002). Using these relations to determine the variations in angular diameter of a Cepheid, and combining with a determination from radial velocity of the variation in the physical diameter, allows the distance of the star to be determined as in the classical Baade-Wesselink method. Such observations are being used to determine distances to members of the local group of galaxies (Gieren et al., 2000; Macri et al., 2001).

A crucial yardstick for the determination of galactic distances is provided by the $R R$ Lyrae stars, and hence it is important to determine the distance to RR Lyrae itself. A much improved trigonometric parallax to this star has been obtained by Benedict et al. (2002a), using FGS on the Hubble Space Telescope. A consistent value was obtained independently from a theoretically predicted period-luminosity relation by Bono et al. (2002). From these results a distance modulus to the Large Magellanic Cloud (LMC) of very near 18.50 is obtained. Benedict et al. (2002b) similarly determined the parallax of $\delta$ Cephei with FGS, yielding a consistent value of the distance modulus of the LMC.

A breakthrough on the pulsational properties of RR Lyrae stars came with the detection by Olech et al. (1999) of evidence for nonradial oscillations in RR Lyrae stars in the globular cluster M55. New observational techniques allowing detailed study of variable stars in members of the local group are improving the statistics for different types of pulsators and substantially extending the range of parameters (particularly metallicity) for which such stars can be investigated. Alcock et al. (2000) and Moskalik \& Poretti (2002) investigated nonradial pulsation in RR Lyrae stars in the LMC and the Galactic Bulge, respectively. Other striking examples are observations of Cepheids in eclipsing binary systems in the LMC (Alcock et al., 2002), as well as the observations of AGB variables (Menzies et al., 2002) and RR Lyrae stars (Held et al., 2001) in the dwarf spheroidal galaxy Leo I. Further advances may be expected from multi-object spectrographs with moderate or high resolution on large telescopes, such as FLAMES on the Very Large Telescope (VLT) of the European Southern Observatory (ESO) (e.g., Bouchy et al., 2002). Variable stars in the local group will be the topic of IAU Colloquium 193 in New Zealand, July 2003.

\section{Compact, Highly Evolved Pulsators}

The study of compact pulsators continues to develop rapidly. An overview of these stars was provided by Fontaine et al. (2002). A substantial number, more than 30 , of pulsating subdwarf B (sdB) stars (or EC 14026 stars) are now known and more continue to be discovered (e.g., Billères et al., 2002). Their pulsations have also been detected in radialvelocity observations (e.g., O'Toole et al., 2000). Their rich spectra of acoustic modes provide excellent opportunities for asteroseismology; for example, the analysis by Brassard et al. (2001) yielded precise and detailed parameters for the star PG0014+67. Very recently, a new class of longer-period pulsators was announced by Green et al. (2002), at slightly lower effective temperature than for the 'classical' pulsating sdB stars; these presumably show g-mode oscillations, with very interesting asteroseismic potential. 
Observations of period changes in white dwarfs offer rare opportunities for studying stellar evolution in action. O'Brien \& Kawaler (2000) analysed the very interesting case of the cooling of pre-white dwarfs, which may be driven by neutrino emission in the core. By constraining models of white-dwarf cooling, observations of period changes are potentially very valuable for the use of white dwarfs for cosmochronology (for a review, see Fontaine et al., 2001). Analysis of the observed periods are providing further constraints on the properties of the interior structure of white dwarfs. As noted by Metcalfe et al. (2001), it may be possible to constrain the ratio between $\mathrm{C}$ and $\mathrm{O}$ in the core of the white dwarfs and hence the rate of the ${ }^{12} \mathrm{C}(\alpha, \gamma)^{16} \mathrm{O}$ reaction which led to that ratio.

\section{B-Star Pulsations}

In the study of B-type main-sequence pulsators, emphasis was mainly put on the gathering of high-resolution spectroscopic time series of bright $\beta$ Cep stars (e.g., Ausseloos et al., 2002) and slowly pulsating B stars (e.g., De Cat et al., 2000; Mathias et al., 2001). Moreover, considerable efforts were made to improve the methods for interpretation of the data (Cugier \& Daszyńska, 2001; Townsend, 2002; De Ridder et al., 2002), with specific attention to nonadiabatic effects in the outer atmosphere (Dupret et al., 2002). The search for hot pulsators in the Magellanic Clouds (Pigulski \& Kołaczkowski, 2002) is important to test in detail the dependence of the excitation on metallicity.

The study of Be stars has a historical significance dating back to the very birth of stellar spectroscopy. Their complex variability nature with different time scales from days to years continued to be revealed during numerous observational studies. The exact cause of the mass ejection remains unsolved. The diagnostic value of these stars should be further explored, as it has become clear from several studies of young clusters that B stars turn Be during the second part of their main-sequence lifetime (e.g., Fabregat \& Torrejón, 2000).

In the uppermost part of the HR diagram, we encounter the microvariable $O B$ supergiants, named S Doradus stars after the prototype. The recent summary of the variability of such stars reveals a wealth of different types of variability (van Genderen, 2001). One encounters the very rare eruptive episodes, slow pulsations on a time scale of years/decades and microvariations on a time scale of weeks/months. Precise light curve characteristics of these stars are hard to derive, in view of the long time scales. Thus the efforts of amateur astronomers are of key importance for the study of the variability of blue supergiants.

\section{A-Star Pulsations}

There have been substantial theoretical developments concerning the interaction of pulsation and magnetic fields in roAp stars (Cunha \& Gough, 2000; Bigot et al., 2000) and the excitation mechanism (Balmforth et al., 2001). Bigot \& Dziembowski (2002) presented an entirely new look at the oblique pulsator model: rather than the modes being axisymmetric with pulsation and magnetic axis aligned, the pulsation axis is inclined to both the magnetic and rotation axes; the modes are complex combinations of spherical harmonics, in many cases resulting in travelling waves looking similar to sectoral modes.

The outstanding problem of the sixth frequency in HR 1217 has been solved. Cunha \& Gough and Bigot et al. found that the reflection of the magnetic and acoustic components of the modes changes with frequency so that the magnetic perturbation to the frequencies can have a large range. These perturbations are progressive over a range of frequencies effectively stretching the large spacing - then as the acoustic and magnetic reflections pass through a full cycle in their relation with each other, there is a big jump in the magnetic perturbation. Cunha (2001) used this to predict an additional frequency in HR 1217 between the fifth and sixth modes and separated from the fifth frequency by about half of the large spacing. Kurtz et al. (2002) found the predicted frequency in a WET campaign, giving strong support to the theory of Cunha.

High-resolution spectra of roAp stars (e.g., Kochukhov \& Ryabchikova, 2001) show very clearly the extreme stratification effects of abundances and the short vertical wavelength of 
the pulsation modes in roAp stars. For $\gamma$ Equ lines of Pr III and Nd III show significant radial velocity variations, while most other lines in the spectrum show none. A plausible interpretation of this phenomenon is that those ions are concentrated in thin layers by radiative diffusion, near a vertical anti-node of the pulsation mode. The line profiles also show clearly the signature of travelling waves. This is qualitatively expected in the improved oblique pulsator model of Bigot \& Dziembowski, but has yet to be tested quantitatively.

The $\gamma$ Dor stars are late-A to early-F main-sequence variables that pulsate in highovertone $\mathrm{g}$ modes with periods of about $8 \mathrm{hr}$ to days (Zerbi \& Kaye, 2002). The latest instability strip for them (Handler \& Shobbrook, 2002) shows them to have some overlap with the coolest $\delta$ Sct stars. Of five $\gamma$ Dor stars that lie in the $\delta$ Sct instability strip, none shows short-period $\delta$ Sct pulsation, suggesting that there may be a complete separation of the two groups. Should hybrid stars that pulsate in both $\mathrm{p}$ modes and $\mathrm{g}$ modes nevertheless be found, they would be rich asteroseismic targets. Multi-site photometric campaigns for $\gamma$ Dor stars are scarce, so there is a dearth of frequency information for them. Poretti et al. (2002) showed light curves for HD 224838 and HD 224945 that make the multi-periodic nature and long periods of these stars beautifully clear. But only the highest-amplitude frequencies in that work are secure; this shows the potential of photometric campaigns on $\gamma$ Dor stars, and the need for intensive multi-site campaigns.

Intensive observational campaigns on $\delta$ Sct stars continue to find over 20 frequencies in some cases (Breger et al., 2002; Handler et al., 2000), but these are complex objects with rapid rotation, core convection and element separation in some cases. Current models find many hundreds of modes to be excited; with rotational splitting, possible differential rotation, possible mixed modes (with g-mode characteristics in the interior and p-mode characteristics at the surface) and uncertain amounts of core overshooting all allowing substantial adjustment of the model frequencies, it is not possible at present to do more than put a few constraints on the stellar structure using the observed frequencies. By potentially detecting smaller-amplitude modes, the upcoming space observations may help the mode identification and hence the asteroseismic analysis of $\delta$ Sct stars. In the case of XX Pyx, the star has been found to be ellipsoidal (Aerts et al., 2002), explaining the failure of previous attempts to model its oscillations.

\section{Solar-Like Oscillations}

Solar-like oscillations in stars near the main sequence, for long regarded as the holy grail of asteroseismology, have now been unambiguously detected thanks in large part to the development of techniques for very stable radial-velocity determination. Following the groundbreaking results of Bedding et al. (2001) for $\beta$ Hyi and the striking observations by Bouchy \& Carrier (2001) of $\alpha$ Cen A, several stars have been found to exhibit solar-like oscillations (see reports in Thompson et al., 2002). The analysis of the so-far mostly singlesite data has been complicated by daily sidelobes, but this undoubtedly marks the birth of asteroseismology based on solar-like oscillations. Extensive data are expected from the space projects to be launched over the next few years. The theoretical interpretation of the data is just beginning. For example, Thévenin et al. (2002) analysed the $\alpha$ Cen system; interestingly, models based on previous astrometric and photometric data on the system were not fully consistent with the frequency observations, perhaps indicating a need for revisions of the modelling. Such tests are obviously an important goal of asteroseismology.

Solar-like oscillations are believed to be excited stochastically by convection and are therefore predicted to be present in all stars with substantial outer convection zones (e.g., Stein \& Nordlund, 2001). Indeed, Frandsen et al. (2002) found oscillations with a power distribution very similar to that of the Sun, but peaking at periods of $3-4$ hours, in the G7 giant $\xi$ Hya. At much longer periods, Christensen-Dalsgaard et al. (2001) showed statistical evidence, based on decades of observations by the AAVSO, that semiregular red-giant variables might be caused by solar-like oscillations, and solar-like characteristics were found by Bedding (2002) for individual stars in this group. In addition to providing information about these stars, the identification of the cause of their variability as being 
solar-like would greatly extend the range of stellar parameters over which this mechanism can be investigated.

Jørgen Christensen-Dalsgaard

President of the Commission

\section{References}

Aerts, C., Bedding, T. R. \& Christensen-Dalsgaard, J., eds, 2002, Proc. IAU Colloq. 185: Radial and nonradial pulsations as probes of stellar physics, ASP Conf. Ser., vol. 259, $i-x x v i i, 1-667$.

Aerts, C., Handler, G., Arentoft, T., Vandenbussche, B., Medupe, R. \& Sterken, C., 2002, $M N R A S, \mathbf{3 3 3}, \mathrm{L} 35$.

Alcock, C., Allsman, R., Alves, D. R., et al., 2000, ApJ, 542, 257.

Alcock, C., Allsman, R. A., Alves, D. R., et al., 2002, ApJ, 573, 338.

Ausseloos, M., Aerts, C., Uytterhoeven, K., Schrijvers, C., Waelkens, C. \& Cuypers, J., 2002, $A \& A, 384,209$.

Baglin, A., Auvergne, M., Barge, P., Buey, J.-T., Catala, C., Michel, E., Weiss, W., and the COROT Team, 2002, in Favata et al. (2002), 17.

Balmforth, N. J., Cunha, M. S., Dolez, N., Gough, D. O. \& Vauclair, S., 2001, MNRAS, 323, 362.

Bedding, T. R., 2002, in Thompson et al. (2002), in press.

Bedding, T. R., Butler, R. P., Kjeldsen, H., Baldry, I. K., O'Toole, S. J., Tinney, C. G., Marcey, G. W., Kienzle, F. \& Carrier, F., 2001, ApJ, 549, L105.

Benedict, G. F., McArthur, B. E., Frederick, L. W., et al., 2002a, $A J, 123,473$.

Benedict, G. F., McArthur, B. E., Frederick, L. W., et al., 2002b, $A J, 124,1695$.

Bigot, L. \& Dziembowski, W. A., 2002, A\&A, 391, 235.

Bigot, L., Provost, J., Berthomieu, G., Dziembowski, W. A. \& Goode, P. R., 2000, $A \mathscr{G A}$, 356, 218.

Billères, M., Fontaine, G., Brassard, P. \& Liebert, J., 2002, ApJ, 578, 515.

Bono, G., Caputo, F., Castellani, V., Marconi, M. \& Storm, J., 2002, MNRAS, 332, L78.

Bouchy, F. \& Carrier, F., 2001, A\&A, 374, L5.

Bouchy, F., Mayor, M., Pepe, F., Queloz, D. \& Udry, S., 2002, in Favata et al. (2002), 153.

Brassard, P., Fontaine, G., Billères, M., Charpinet, S., Liebert, J. \& Saffer, R. A., 2001, ApJ, 563, 1013.

Breger, M., Garrido, R., Handler, G., et al., 2002, MNRAS, 329. 531.

Buzasi, D., Catanzarite, J., Laher, R., et al., 2000, $A p J$, 532, L133.

Christensen-Dalsgaard, J., 2002, in Favata et al. (2002), 25.

Christensen-Dalsgaard, J., Kjeldsen, H. \& Mattei, J. A., 2001, ApJ, 562, L141.

Cugier, H. \& Daszyńska, J., 2001, $A \& A, \mathbf{3 7 7}, 113$.

Cunha, M. S., 2001, MNRAS, 325, 373.

Cunha, M. S. \& Gough, D., 2000, MNRAS, 319, 1020.

Cuypers, J., Aerts, C., Buzasi, D., Catanzarite, J., Conrow, T. \& Laher, R., 2002, $A \& A$, 392, 599.

De Cat, P., Aerts, C., De Ridder, J., Kolenberg, K., Meeus, G. \& Decin, L., 2000, $A \& A$, 355, 1015.

De Ridder, J., Dupret, M.-A., Neuforge, C. \& Aerts, C., 2002, A\&A, 385, 572.

Dupret, M.-A., De Ridder, J., Neuforge, C., Aerts, C. \& Scuflaire, R., 2002, $A \mathscr{E} A, \mathbf{3 8 5}$, 563. 
Fabregat, J. \& Torrejón, J. M., 2000, $A \& A, 357,451$.

Favata, F., 2002, in Favata et al. (2002), 3.

Favata, F., Roxburgh, I. W. \& Galadí-Enríquez, P., eds, 2002, Proc. 1st Eddington Workshop, 'Stellar Structure and Habitable Planet Finding', ESA SP-485, ESA Publications Division, Noordwijk, The Netherlands, pp. $i-x i, 1-366$.

Fontaine, G., Brassard, P. \& Bergeron, P., 2001, PASP, 113, 409.

Fontaine, G., Brassard, P. \& Charpinet, S., 2002, in Thompson et al. (2002), in press.

Frandsen, S., Carrier, F., Aerts, C., et al., 2002, A\&GA, 394, L5.

Gieren, W. P., Storm, J., Fourqué, P., Mennickent, R. E. \& Gómez, M., 2000, ApJ, 533, L107.

Green, E. M., Callerame, K., Seitenzahl, I. R., et al., 2002, in Thompson et al. (2002), in press.

Handler, G. \& Shobbrook, R. R., 2002, MNRAS, 333, 251.

Handler, G., Arentoft, T., Shobbrook, R. R., et al., 2000, MNRAS, 318, 511.

Held, E. V., Clementini, G., Rizzi, L., Momany, Y., Saviane, I. \& Di Fabrizio, L., 2001, ApJ, 562, L39.

Kochukhov, O. \& Ryabchikova, T., 2001, A\&GA, 374, 615.

Kurtz, D. W., Kawaler, S. D., Riddle, R. L., et al., 2002, MNRAS, 330, L57.

Lane, B. F., Creech-Eakman, M. J. \& Nordgren, T. E., 2002, ApJ, 573, 330.

Macri, L. M., Stanek, K. Z., Sasselov, D. D. \& Krockenberger, M., 2001, AJ, 121, 870.

Mathias, P., Aerts, C., Briquet, M., De Cat, P., Cuypers, J., Van Winckel, H., \& Le Contel, J. M., 2001, $A \mathscr{E} A, \mathbf{3 7 9}, 905$.

Matthews, J., Kuschnig, R. \& Shkolnik, E., 2001, in Helio- and Asteroseismology at the Dawn of the Millennium: Proc. SOHO 10/GONG 2000 Workshop, ESA SP-464, ESA Publications Division, Noordwijk, The Netherlands, 385.

Menzies, J., Feast, M., Tanabé, T., Whitelock, P. \& Nakada, Y., 2002, MNRAS, 335, 923.

Metcalfe, T. S., Winget, D. E. \& Charbonneau, P., 2001, ApJ, 557, 1021.

Moskalik, P. \& Poretti, E., 2002, , in Aerts et al. (2002), 392.

Nordgren, T. E., Lane, B. F., Hindsley, R. B. \& Kervella, P., 2002, $A J$, 123, 3380.

O'Brien, M. S. \& Kawaler, S. D., 2000, ApJ, 539, 372.

Olech, A., Kaluzny, J., Thompson, I. B., Pych, W., Krzeminski, W. \& SchwarzenbergCzerny, A., 1999, $A J, 118,442$.

O'Toole, S. J., Bedding, T. R., Kjeldsen, H., Teixeira, T. C., Roberts, G., van Wyk, F., Kilkenny, D., D'Cruz, N. \& Baldry, I. K., 2000, ApJ, 537, L53.

Pigulski, A. \& Kołaczkowski, Z., 2002, $A \& A$, 388, 88.

Poretti, E., Koen, C., Bossi, M., Rodríguez, E., Martín, S., Krisciunas, K., Akan, M. C., Crowe, R., Wilcox, M., Ibanoglu, C. \& Evren, S., 2002, $A \& A, \mathbf{3 8 4}, 513$.

Stein, R. F. \& Nordlund, A., 2001, ApJ, 546, 585.

Thévenin, F., Provost, J., Morel, P., Berthomieu, G., Bouchy, F. \& Carrier, F., 2002, $A \& A$, 392, L9.

Thompson, M. J., Cunha, M. S. \& Monteiro, M. J. P. F. G., eds, 2002, Asteroseismology across the HR diagram, Kluwer Academic Publishers, Dordrecht, in press.

Townsend, R. H. D., 2002, MNRAS, 330, 855.

Udalski, A., Soszyński, I., Szymański, M., Kubiak, M., Pietrzyński, G., Woźniak, P. \& Żebruń, K., 1999, Acta Astron., 49, 1.

van Genderen, A. M., 2001, $A \& A, \mathbf{3 6 6}, 508$.

Zerbi, F. M. \& Kaye, A. B., 2002, in Aerts et al. (2002), 494. 